\title{
The Computation of $\pi$ to 29,360,000 Decimal Digits Using Borweins' Quartically Convergent Algorithm
}

\author{
By David H. Bailey
}

\begin{abstract}
In a recent work [6], Borwein and Borwein derived a class of algorithms based on the theory of elliptic integrals that yield very rapidly convergent approximations to elementary constants. The author has implemented Borweins' quartically convergent algorithm for $1 / \pi$, using a prime modulus transform multi-precision technique, to compute over 29,360,000 digits of the decimal expansion of $\pi$. The result was checked by using a different algorithm, also due to the Borweins, that converges quadratically to $\pi$. These computations were performed as a system test of the Cray-2 operated by the Numerical Aerodynamical Simulation (NAS) Program at NASA Ames Research Center. The calculations were made possible by the very large memory of the Cray-2.

Until recently, the largest computation of the decimal expansion of $\pi$ was due to Kanada and Tamura [12] of the University of Tokyo. In 1983 they computed approximately 16 million digits on a Hitachi S-810 computer. Late in 1985 Gosper [9] reported computing 17 million digits using a Symbolics workstation. Since the computation described in this paper was performed, Kanada has reported extending the computation of $\pi$ to over 134 million digits (January 1987).

This paper describes the algorithms and techniques used in the author's computation, both for converging to $\pi$ and for performing the required multi-precision arithmetic. The results of statistical analyses of the computed decimal expansion are also included.
\end{abstract}

1. Introduction. The computation of the numerical value of the constant $\pi$ has been pursued for centuries for a variety of reasons, both practical and theoretical. Certainly, a value of $\pi$ correct to 10 decimal places is sufficient for most "practical" applications. Occasionally, there is a need for double-precision or even multi-precision computations involving $\pi$ and other elementary constants and functions in order to compensate for unusually severe numerical difficulties in an extended computation. However, the author is not aware of even a single case of a "practical" scientific computation that requires the value of $\pi$ to more than about 100 decimal places.

Beyond immediate practicality, the decimal expansion of $\pi$ has been of interest to mathematicians, who have still not been able to resolve the question of whether the digits in the expansion of $\pi$ are "random". In particular, it is widely suspected that the decimal expansions of $\pi, e, \sqrt{2}, \sqrt{2 \pi}$, and a host of related mathematical constants all have the property that the limiting frequency of any digit is one tenth, and that the limiting frequency of any $n$-long string of digits is $10^{-n}$. Such a guaranteed property could, for instance, be the basis of a reliable pseudo-random number generator. Unfortunately, this assertion has not been proven in even one instance. Thus, there is a continuing interest in performing statistical analyses on

Received January 27, 1986; revised April 27, 1987.

1980 Mathematics Subject Classification (1985 Revision). Primary 11-04, 65-04. 
the decimal expansions of these numbers to see if there is any irregularity that would suggest this assertion is false.

In recent years, the computation of the expansion of $\pi$ has assumed the role as a standard test of computer integrity. If even one error occurs in the computation, then the result will almost certainly be completely in error after an initial correct section. On the other hand, if the result of the computation of $\pi$ to even 100,000 decimal places is correct, then the computer has performed billions of operations without error. For this reason, programs that compute the decimal expansion of $\pi$ are frequently used by both manufacturers and purchasers of new computer equipment to certify system reliability.

2. History. The first serious attempt to calculate an accurate value for the constant $\pi$ was made by Archimedes, who approximated $\pi$ by computing the areas of equilateral polygons with increasing numbers of sides. More recently, infinite series have been used. In 1671 Gregory discovered the arctangent series

$$
\tan ^{-1}(x)=x-\frac{x^{3}}{3}+\frac{x^{5}}{5}-\frac{x^{7}}{7}+\cdots
$$

This discovery led to a number of rapidly convergent algorithms. In 1706 Machin used Gregory's series coupled with the identity

$$
\pi=16 \tan ^{-1}(1 / 5)-4 \tan ^{-1}(1 / 239)
$$

to compute 100 digits of $\pi$.

In the nearly 300 years since that time, most computations of the value of $\pi$, even those performed by computer, have employed some variation of this technique. For instance, a series based on the identity

$$
\pi=24 \tan ^{-1}(1 / 8)+8 \tan ^{-1}(1 / 57)+4 \tan ^{-1}(1 / 239)
$$

was used in a computation of $\pi$ to 100,000 decimal digits using an IBM 7090 in 1961 [15]. Readers interested in the history of the computation $\pi$ are referred to Beckmann's entertaining book on the subject [2].

3. New Algorithms for $\pi$. Only very recently have algorithms been discovered that are fundamentally faster than the above techniques. In 1976 Brent [7] and Salamin [14] independently discovered an approximation algorithm based on elliptic integrals that yields quadratic convergence to $\pi$. With all of the previous techniques, the number of correct digits increases only linearly with the number of iterations performed. With this new algorithm, each additional iteration of the algorithm approximately doubles the number of correct digits. Kanada and Tamura employed this algorithm in 1983 to compute $\pi$ to over 16 million decimal digits.

More recently, J. M. Borwein and P. B. Borwein [4] discovered another quadratically convergent algorithm for $\pi$, together with similar algorithms for fast computation of all the elementary functions. Their quadratically convergent algorithm for $\pi$ can be stated as follows: Let $a_{0}=\sqrt{2}, b_{0}=0, p_{0}=2+\sqrt{2}$. Iterate

$$
a_{k+1}=\frac{\left(\sqrt{a_{k}}+1 / \sqrt{a_{k}}\right)}{2}, \quad b_{k+1}=\frac{\sqrt{a_{k}}\left(1+b_{k}\right)}{a_{k}+b_{k}}, \quad p_{k+1}=\frac{p_{k} b_{k+1}\left(1+a_{k+1}\right)}{1+b_{k+1}} .
$$

Then $p_{k}$ converges quadratically to $\pi$ : Successive iterations of this algorithm yield $3,8,19,40,83,170,345,694,1392$, and 2788 correct digits of the expansion of $\pi$. 
However, it should be noted that this algorithm is not self-correcting for numerical errors, so that all iterations must be performed to full precision. In other words, in a computation of $\pi$ to 2788 decimal digits using the above algorithm, each of the ten iterations must be performed with more than 2788 digits of precision.

Most recently, the Borweins [6] have discovered a general technique for obtaining even higher-order convergent algorithms for certain elementary constants. Their quartically convergent algorithm for $1 / \pi$ can be stated as follows: Let $a_{0}=6-4 \sqrt{2}$ and $y_{0}=\sqrt{2}-1$. Iterate

$$
\begin{aligned}
& y_{k+1}=\frac{1-\left(1-y_{k}^{4}\right)^{1 / 4}}{1+\left(1-y_{k}^{4}\right)^{1 / 4}} \\
& a_{k+1}=a_{k}\left(1+y_{k+1}\right)^{4}-2^{2 k+3} y_{k+1}\left(1+y_{k+1}+y_{k+1}^{2}\right) .
\end{aligned}
$$

Then $a_{k}$ converges quartically to $1 / \pi$ : Each successive iteration approximately quadruples the number of correct digits in the result. As in the previous case, each iteration must be performed to at least the level of precision desired for the final result.

4. Multi-Precision Arithmetic Techniques. A key element of a very high precision computation of this sort is a set of high-performance routines for performing multi-precision arithmetic. A naive approach to multi-precision computation would require a prohibitive amount of processing time and would, as a result, sharply increase the probability that an undetected hardware error would occur, rendering the result invalid. In addition to employing advanced algorithms for such key operations as multi-precision multiplication, it is imperative that these algorithms be implemented in a style that is conducive for high-speed computation on the computer being used.

The computer used for these computations is the Cray-2 at the NASA Ames Research Center. This computation was performed to test the integrity of the Cray-2 hardware, as well as the Fortran compiler and the operating system. The Cray-2 is particularly well suited for this computation because of its very large main memory, which holds $2^{28}=268,435,456$ words (one word is 64 bits of data). With this huge capacity, all data for these computations can be contained entirely within main memory, insuring ease of programming and fast execution.

No attempt was made to employ more than one of the four central processing units in the Cray-2. Thus, at the same time these calculations were being performed, the computer was executing other jobs on the other processors. However, full advantage was taken of the vector operations and vector registers of the system. Considerable care was taken in programming to insure that the multi-precision algorithms were implemented in a style that would admit vector processing. Most key loops were automatically vectorized by the Cray-2 Fortran compiler. For those few that were not automatically vectorized, compiler directives were inserted to force vectorization. As a result of this effort, virtually all arithmetic operations were performed in vector mode, which on the Cray-2 is approximately 20 times faster than scalar mode. Because of the high level of vectorization that was achieved using the Fortran compiler, it was not necessary to use assembly language, nonstandard constructs, or library subroutines. 
A multi-precision number is represented in these computations as an $(n+2)$ long array of floating-point whole numbers. The first cell contains the sign of the number, either $1,-1$, or 0 (reserved for an exact zero). The second cell of the array contains the exponent (powers of the radix), and the remaining $n$ cells contain the mantissa. The radix selected for the multi-precision numbers is $10^{7}$. Thus the number 1.23456789 is represented by the array $1,0,1,2345678,9000000,0,0, \ldots, 0$.

A floating-point representation was chosen instead of an integer representation because the hardware of numerical supercomputers such as the Cray-2 is designed for floating-point computation. Indeed, the Cray-2 does not even have full-word integer multiply or divide hardware instructions. Such operations are performed by first converting the operands to floating-point form, using the floating-point unit, and converting the results back to fixed-point (integer) form. A decimal radix was chosen instead of a binary value because multiplications and divisions by powers of two are not performed any faster than normal on the Cary-2 (in vector mode). Since a decimal radix is clearly preferable to a binary radix for program troubleshooting and for input and output, a decimal radix was chosen. The value $10^{7}$ was chosen because it is the largest power of ten that will fit in half of the mantissa of a single word. In this way two of these numbers may be multiplied to obtain the exact product using ordinary single-precision arithmetic.

Multi-precision addition and subtraction are not computationally expensive compared to multiplication, division, and square root extraction. Thus, simple algorithms suffice to perform addition and subtraction. The only part of these operations that is not immediately conducive to vector processing is releasing the carries for the final result. This is because the normal "schoolboy" approach of beginning at the last cell and working forward is a recursive operation. On a vector supercomputer this is better done by starting at the beginning and releasing the carry only one cell back for each cell processed. Unfortunately, it cannot be guaranteed that one application of this process will release all carries (consider the case of two or more consecutive 9999999's, followed by a number exceeding $10^{7}$ ). Thus it is necessary to repeat this operation until all carries have been released (usually one or two additional times). In the rare cases where three applications of this vectorized process are not successful in releasing all carries, the author's program resorts to the scalar "schoolboy" method.

Provided a fast multi-precision multiplication procedure is available, multiprecision division and square root extraction may be performed economically using Newton's iteration, as follows. Let $x_{0}$ and $y_{0}$ be initial approximations to the reciprocal of $a$ and to the reciprocal of the square root of $a$, respectively. Then

$$
x_{k+1}=x_{k}\left(2-a x_{k}\right), \quad y_{k+1}=\frac{y_{k}\left(3-a y_{k}^{2}\right)}{2}
$$

both converge quadratically to the desired values. One additional full-precision multiplication yields the quotient and the square root, respectively. What is especially attractive about these algorithms is that the first iteration may be performed using ordinary single-precision arithmetic, and subsequent iterations may be performed using a level of precision that approximately doubles each time. Thus the total cost of computation is only about twice the cost of the final iteration, plus the one additional multiplication. As a result, a multi-precision division costs only about 
five times as much as a multi-precision multiplication, and a multi-precision square root costs only about seven times as much as a multi-precision multiplication.

5. Multi-Precision Multiplication. It can be seen from the above that the key component of a high-performance multi-precision arithmetic system is the multiply operation. For modest levels of precision (fewer than about 1000 digits), some variation of the usual "schoolboy" method is sufficient, although care must be taken in the implementation to insure that the operations are vectorizable. Above this level of precision, however, other more sophisticated techniques have a significant advantage. The history of the development of high-performance multiply algorithms will not be reviewed here. The interested reader is referred to Knuth [13]. It will suffice to note that all of the current state-of-the-art techniques derive from the following fact of Fourier analysis: Let $F(x)$ denote the discrete Fourier transform of the sequence $x=\left(x_{0}, x_{1}, x_{2}, \ldots, x_{N-1}\right)$, and let $F^{-1}(x)$ denote the inverse discrete Fourier transform of $x$ :

$$
F_{k}(x)=\sum_{j=0}^{N-1} x_{j} \omega^{j k}, \quad F_{k}^{-1}(x)=\frac{1}{N} \sum_{j=0}^{N-1} x_{j} \omega^{-j k}
$$

where $\omega=e^{-2 \pi i / N}$ is a primitive $N$ th root of unity. Let $C(x, y)$ denote the convolution of the sequences $x$ and $y$ :

$$
C_{k}(x, y)=\sum_{j=0}^{N-1} x_{j} y_{k-j}
$$

where the subscript $k-j$ is to be interpreted as $k-j+N$ if $k-j$ is negative. Then the "convolution theorem", whose proof is a straightforward exercise, states that

$$
F[C(x, y)]=F(x) F(y),
$$

or expressed another way,

$$
C(x, y)=F^{-1}[F(x) F(y)]
$$

This result is applicable to multi-precision multiplication in the following way. Let $x$ and $y$ be $n$-long representations of two multi-precision numbers (without the sign or exponent words). Extend $x$ and $y$ to length $2 n$ by appending $n$ zeros at the end of each. Then the multi-precision product $z$ of $x$ and $y$, except for releasing the carries, can be written as follows:

$$
\begin{aligned}
z_{0} & =x_{0} y_{0} \\
z_{1} & =x_{0} y_{1}+x_{1} y_{0} \\
z_{2} & =x_{0} y_{2}+x_{1} y_{1}+x_{2} y_{0} \\
& \vdots \\
z_{n-1} & =x_{0} y_{n-1}+x_{1} y_{n-2}+\cdots+x_{n-1} y_{0} \\
& \vdots \\
z_{2 n-3} & =x_{n-1} y_{n-2}+x_{n-2} y_{n-1} \\
z_{2 n-2} & =x_{n-1} y_{n-1} \\
z_{2 n-1} & =0 .
\end{aligned}
$$


It can now be seen that this "multiplication pyramid" is precisely the convolution of the two sequences $x$ and $y$, where $N=2 n$. The convolution theorem states that the multiplication pyramid can be obtained by performing two forward discrete Fourier transforms, one vector complex multiplication, and one reverse transform, each of length $N=2 n$. Once the resulting complex numbers have been rounded to the nearest integer, the final multi-precision product may be obtained by merely releasing the carries as described in the section above on addition and subtraction.

The key computational savings here is that the discrete Fourier transform may of course be economically computed using some variation of the "fast Fourier transform" (FFT) algorithm. It is most convenient to employ the radix two fast Fourier transform since there is a wealth of literature on how to efficiently implement this algorithm (see [1], [8], and [16]). Thus, it will be assumed from this point that $N=2^{m}$ for some integer $m$.

One useful "trick" can be employed to further reduce the computational requirement for complex transforms. Note that the input data vectors $x$ and $y$ and the result vector $z$ are purely real. This fact can be exploited by using a simple procedure ([8, p. 169]) for performing real-to-complex and complex-to-real transforms that obtains the result with only about half the work otherwise required.

One important item has been omitted from the above discussion. If the radix $10^{7}$ is used, then the product of two cells will be in the neighborhood of $10^{14}$, and the sum of a large number of these products cannot be represented exactly in the 48bit mantissa of a Cray-2 floating-point word. In this case the rounding operation at the completion of the transform will not be able to recover the exact whole number result. As a result, for the complex transform method to work correctly, it is necessary to alter the above scheme slightly. The simplest solution is to use the radix $10^{6}$ and to divide all input data into two words with only three digits each. Although this scheme greatly increases the memory space required, it does permit the complex transform method to be used for multi-precision computation up to several million digits on the Cray-2.

6. Prime Modulus Transforms. Some variation of the above method has been used in almost all high-performance multi-precision computer programs, including the program used by Kanada and Tamura. However, it appears to break down for very high-precision computation (beyond about ten million digits on the Cray-2), due to the round-off error problem mentioned above. The input data can be further divided into two digits per word or even one digit per word, but only with a substantial increase in run time and main memory. Since a principal goal in this computation was to remain totally within the Cray-2 main memory, a somewhat different method was used.

It can readily be seen that the technique of the previous section, including the usage of a fast Fourier transform algorithm, can be applied in any number field in which there exists a primitive $N$ th root of unity $\omega$. This requirement holds for the field of the integers modulo $p$, where $p$ is a prime of the form $p=k N+1([11, \mathrm{p}$. 85]). One significant advantage of using a prime modulus field instead of the field of complex numbers is that there is no need to worry about round-off error in the results, since all computations are exact. 
However, there are some difficulties in using a prime modulus field for the transform operations above. The first is to find a prime $p$ of the form $k N+1$, where $N=2^{m}$. The second is to find a primitive $N$ th root of unity modulo $p$. As it turns out, it is not too hard using a computer to find both of these numbers by direct search. Thirdly, one must compute the multiplicative inverse of $N$ modulo $p$. This can be done using a variation of the Euclidean algorithm from elementary number theory. Note that each of these calculations needs to be performed one time only.

A more troublesome difficulty in using a prime modulus transform is the fact that the final multiplication pyramid results are only recovered modulo $p$. If $p$ is greater than about $10^{24}$ then this is not a problem, but the usage of such a large prime would require quadruple-precision arithmetic operations to be performed in the inner loop of the fast Fourier transform, which would very greatly increase the run time. A simpler and faster approach to the problem is to use two primes, $p_{1}$ and $p_{2}$, each slightly greater than $10^{12}$, and to perform the transform algorithm above using each prime. Then the Chinese remainder theorem may be applied to the results modulo $p_{1}$ and $p_{2}$ to obtain the results modulo the product $p_{1} p_{2}$. Since $p_{1} p_{2}$ is greater than $10^{24}$, these results will be the exact multiplication pyramid numbers. Unfortunately, double-precision arithmetic must still be performed in the fast Fourier transform and in the Chinese remainder theorem calculation. However, the whole-number format of the input data simplifies these operations, and it is possible to program them in a vectorizable fashion.

Borodin and Munro ([3, p. 90]) have suggested using three transforms with three primes $p_{1}, p_{2}$ and $p_{3}$, each of which is just smaller than half of the mantissa, and using the Chinese remainder theorem to recover the results modulo $p_{1} p_{2} p_{3}$. In this way, double-precision operations are completely avoided in the inner loop of the FFT. This scheme runs quite fast, but unfortunately the largest transform that can be performed on the Cray-2 using this system is $N=2^{19}$, which corresponds to a maximum precision of about three million digits.

Readers interested in studying about prime modulus number fields, the Euclidean algorithm, or the Chinese remainder theorem are referred to any elementary text on number theory, such as [10] or [11]. Knuth [13] and Borodin [3] also provide excellent information on using these tools for computation.

7. Computational Results. The author has implemented all three of the above techniques for multi-precision multiplication on the Cray-2. By employing special high-performance techniques [1], the complex transform can be made to run the fastest, about four times faster than the two-prime transform method. However, the memory requirement of the two-prime scheme is significantly less than either the three-prime or the complex scheme, and since the two-prime scheme permits very high-precision computation, it was selected for the computations of $\pi$.

One of the author's computations used twelve iterations of Borweins' quartic algorithm for $1 / \pi$, followed by a reciprocal operation, to yield $29,360,128$ digits of $\pi$. In this computation, approximately 12 trillion arithmetic operations were performed. The run took 28 hours of processing time on one of the four Cray-2 central processing units and used 138 million words of main memory. It was started on January 7, 1986 and completed January 9, 1986. The program was not running this entire time--the system was taken down for service several times, and the run 
was frequently interrupted by other programs. Restarting the computation after a system down was a simple matter since the two key multi-precision number arrays were saved on disk after the completion of each iteration.

This computation was checked using 24 iterations of Borweins' quadratically convergent algorithm for $\pi$. This run took 40 hours processing time and 147 million words of main memory. A comparison of these output results with the first run found no discrepancies except for the last 24 digits, a normal truncation error. Thus it can be safely assumed that at least 29,360,000 digits of the final result are correct.

It was discovered after both computations were completed that one loop in the Chinese remainder theorem computation was inadvertently performed in scalar mode instead of vector mode. As a result, both of these calculations used about $25 \%$ more run time than would otherwise have been required. This error, however, did not affect the validity of the computed decimal expansions.

8. Statistical Analysis of $\pi$. Probably the most significant mathematical motivation for the computation of $\pi$, both historically and in modern times, has been to investigate the question of the randomness of its decimal expansion. Before Lambert proved in 1766 that $\pi$ is irrational, there was great interest in checking whether or not its decimal expansion eventually repeats, thus disclosing that $\pi$ is rational. Since that time there has been a continuing interest in the still unanswered question of whether the expansion is statistically random. It is of course strongly suspected that the decimal expansion of $\pi$, if computed to sufficiently high precision, will pass any reasonable statistical test for randomness. The most frequently mentioned conjecture along this line is that any sequence of $n$ digits occurs with a limiting frequency of $10^{-n}$.

With 29,360,000 digits, the frequencies of $n$-long strings may be studied for randomness for $n$ as high as six. Beyond that level the expected number of any one string is too low for statistical tests to be meaningful. The results of tabulated frequencies for one and two digit strings are listed in Tables 1 and 2 . In the first table the $Z$-score numbers are computed as the deviation from the mean divided by the standard deviation, and thus these statistics should be normally distributed with mean zero and variance one.

TABLE 1

Single digit statistics

\begin{tabular}{ccrr}
\hline Digit & Count & Deviation & $Z$-score \\
\hline 0 & 2935072 & -928 & -0.5709 \\
1 & 2936516 & 516 & 0.3174 \\
2 & 2936843 & 843 & 0.5186 \\
3 & 2935205 & -795 & -0.4891 \\
4 & 2938787 & 2787 & 1.7145 \\
5 & 2936197 & 197 & 0.1212 \\
6 & 2935504 & -496 & -0.3051 \\
7 & 2934083 & -1917 & -1.1793 \\
8 & 2935698 & -302 & -0.1858 \\
9 & 2936095 & 95 & 0.0584 \\
\hline
\end{tabular}


TABLE 2

Two digit frequency counts

\begin{tabular}{llllllllll}
\hline 00 & 293062 & 01 & 293970 & 02 & 293533 & 03 & 292893 & 04 & 294459 \\
05 & 294189 & 06 & 292688 & 07 & 292707 & 08 & 294260 & 09 & 293311 \\
10 & 294503 & 11 & 293409 & 12 & 293591 & 13 & 294285 & 14 & 294020 \\
15 & 293158 & 16 & 293799 & 17 & 293020 & 18 & 293262 & 19 & 293469 \\
20 & 293952 & 21 & 293226 & 22 & 293844 & 23 & 293382 & 24 & 293869 \\
25 & 293721 & 26 & 293655 & 27 & 293969 & 28 & 293320 & 29 & 293905 \\
30 & 293718 & 31 & 293542 & 32 & 293272 & 33 & 293422 & 34 & 293178 \\
35 & 293490 & 36 & 293484 & 37 & 292694 & 38 & 294152 & 39 & 294253 \\
40 & 294622 & 41 & 294793 & 42 & 293863 & 43 & 293041 & 44 & 293519 \\
45 & 293998 & 46 & 294418 & 47 & 293616 & 48 & 293296 & 49 & 293621 \\
50 & 292736 & 51 & 294272 & 52 & 293614 & 53 & 293215 & 54 & 293569 \\
55 & 294194 & 56 & 293260 & 57 & 294152 & 58 & 293137 & 59 & 294048 \\
60 & 293842 & 61 & 293105 & 62 & 294187 & 63 & 293809 & 64 & 293463 \\
65 & 293544 & 66 & 293123 & 67 & 293307 & 68 & 293602 & 69 & 293522 \\
70 & 292650 & 71 & 294304 & 72 & 293497 & 73 & 293761 & 74 & 293960 \\
75 & 293199 & 76 & 293597 & 77 & 292745 & 78 & 293223 & 79 & 293147 \\
80 & 292517 & 81 & 292986 & 82 & 293637 & 83 & 294475 & 84 & 294267 \\
85 & 293600 & 86 & 293786 & 87 & 293971 & 88 & 293434 & 89 & 293025 \\
90 & 293470 & 91 & 292908 & 92 & 293806 & 93 & 292922 & 94 & 294483 \\
95 & 293104 & 96 & 293694 & 97 & 293902 & 98 & 294012 & 99 & 293794 \\
\hline
\end{tabular}

The most appropriate statistical procedure for testing the hypothesis that the empirical frequencies of $n$-long strings of digits are random is the $\chi^{2}$ test. The $\chi^{2}$ statistic of the $k$ observations $X_{1}, X_{2}, \ldots, X_{k}$ is defined as

$$
\chi^{2}=\sum_{i=1}^{k} \frac{\left(X_{i}-E_{i}\right)^{2}}{E_{i}}
$$

where $E_{i}$ is the expected value of the random variable $X_{i}$. In this case $k=10^{n}$ and $E_{i}=10^{-n} d$ for all $i$, where $d=29,360,000$ denotes the number of digits. The mean of the $\chi^{2}$ statistic in this case is $k-1$ and its standard deviation is $\sqrt{2(k-1)}$. Its distribution is nearly normal for large $k$. The results of the $\chi^{2}$ analysis are shown in Table 3.

TABLE 3

\begin{tabular}{|c|c|c|}
\hline \multicolumn{3}{|c|}{ Multiple digit $\chi^{2}$ statistics } \\
\hline Length & $\chi^{2}$ value & $Z$-score \\
\hline 1 & 4.869696 & -0.9735 \\
\hline 2 & 84.52604 & -1.0286 \\
\hline 3 & 983.9108 & -0.3376 \\
\hline 4 & 10147.258 & 1.0484 \\
\hline 5 & 100257.92 & 0.5790 \\
\hline 6 & 1000827.7 & 0.5860 \\
\hline
\end{tabular}

Another test that is frequently performed on long pseudo-random sequences is an analysis to check whether the number of $n$-long repeats for various $n$ is within statistical bounds of randomness. An $n$-long repeat is said to occur if the $n$-long 
digit sequence beginning at two different positions is the same. The mean $M$ and the variance $V$ of the number of $n$-long repeats in $d$ digits are (to an excellent approximation)

$$
M=\frac{10^{-n} d^{2}}{2}, \quad V=\frac{11 \cdot 10^{-n} d^{2}}{18} .
$$

Tabulation of repeats in the expansion of $\pi$ was performed by packing the string beginning at each position into a single Cray-2 word, sorting the resulting array, and counting equal contiguous entries in the sorted list. The results of this analysis are shown in Table 4.

\section{TABLE 4}

\begin{tabular}{rrrr}
\multicolumn{4}{c}{ Long repeat statistics } \\
\hline 10 & 42945 & 43100. & -0.677 \\
11 & 4385 & 4310. & 1.033 \\
12 & 447 & 431. & 0.697 \\
13 & 48 & 43.1 & 0.675 \\
14 & 6 & 4.31 & 0.736 \\
15 & 1 & 0.43 & 0.784 \\
\hline
\end{tabular}

A third test frequently performed as a test for randomness is the runs test. This test compares the observed frequency of long runs of a single digit with the number of such occurrences that would be expected at random. The mean and variance of this statistic are the same as the formulas for repeats, except that $d^{2}$ is replaced by $2 d$. Table 5 lists the observed frequencies of runs for the calculated expansion of $\pi$.

The frequencies of long runs are all within acceptable limits of randomness. The only phenomenon of any note in Table 5 is the occurrence of a 9-long run of sevens. However, there is a $29 \%$. chance that a 9-long run of some digit would occur in $29,360,000$ digits, so this instance by itself is not remarkable.

TABLE 5

\begin{tabular}{cccccc}
\multicolumn{8}{c}{ Single-digit run counts } \\
\hline \multicolumn{8}{c}{ Length } & of & Run \\
Digit & 5 & 6 & 7 & 8 & 9 \\
\hline 0 & 308 & 29 & 3 & 0 & 0 \\
1 & 281 & 21 & 1 & 0 & 0 \\
2 & 272 & 23 & 0 & 0 & 0 \\
3 & 266 & 26 & 5 & 0 & 0 \\
4 & 296 & 40 & 6 & 1 & 0 \\
5 & 292 & 30 & 4 & 0 & 0 \\
6 & 316 & 33 & 3 & 0 & 0 \\
7 & 315 & 37 & 6 & 2 & 1 \\
8 & 295 & 36 & 3 & 0 & 0 \\
9 & 306 & 40 & 7 & 0 & 0 \\
\hline
\end{tabular}

9. Conclusion. The statistical analyses that have been performed on the expansion of $\pi$ to $29,360,000$ decimal places have not disclosed any irregularity. The observed frequencies of $n$-long strings of digits for $n$ up to 6 are entirely unremarkable. The numbers of long repeating strings and single-digit $r$ 'ns are completely 
acceptable. Thus, based on these tests, the decimal expansion of $\pi$ appears to be completely random.

\section{Appendix}

Selected Output Listing

Initial 1000 digits:

3 .

14159265358979323846264338327950288419716939937510 58209749445923078164062862089986280348253421170679 82148086513282306647093844609550582231725359408128 48111745028410270193852110555964462294895493038196 44288109756659334461284756482337867831652712019091 45648566923460348610454326648213393607260249141273 72458700660631558817488152092096282925409171536436 78925903600113305305488204665213841469519415116094 33057270365759591953092186117381932611793105118548 07446237996274956735188575272489122793818301194912 98336733624406566430860213949463952247371907021798 60943702770539217176293176752384674818467669405132 00056812714526356082778577134275778960917363717872 14684409012249534301465495853710507922796892589235 42019956112129021960864034418159813629774771309960 51870721134999999837297804995105973173281609631859 50244594553469083026425223082533446850352619311881 71010003137838752886587533208381420617177669147303 59825349042875546873115956286388235378759375195778 18577805321712268066130019278766111959092164201989

Digits $4,999,001$ to $5,000,000$ :

49480754784558100182731931632488412804488722296956 79855015464855780486736535227902836997918084867230 64962221004527085768335035212069684801817137616329 97561738425160340472537100056351640342162492027179 66824926458930960182645026923102266570541641475347 20341554913770421505764452807809035248393621093031 02288096238486877923145240841637271180953058890040 68843766781431498914299893621278545260143140439048 49938801556336059513116731891132765777881364690708 47036863411196323063886507480852125682842257852524 03086993703255692093960818587414181230484153204049 20234989002732447593020323794790776444752398445514 67304403210968985244961967143433964895893190552338 49818852746844924836314634250006421630628686858848 27453318669926734730642735036364002856022218966350 11429182634319974163253372368798553451111253055262 39104082639970934508146672521381105913047210052428 18988626533169469331951675296209306752291590715999 89846179288059262000848638138811280944056488021060 48865855191846702365421761783505181721320764619715 
Digits $9,999,001$ to $10,000,000$ :

55097818243516728227849910720400286757907904466335 12718202979525150617725334066894988956424703269230 15399820900390166275224338184424808589395293652582 53635658584175485536744818650289245188206447853280 79129675504865572929083083485483937583334671019089 12067114536955173140929461823466478725289529974204 02127635235923293305770179423865225963240694027480 60412880303092452481034941582735932443887273109397 41634889604695819245395151341043433998381874650972 33692635225791472454244401326312964396391209607800 16344851199125420819737407446045899742145731042313 64456486501937801063526603744056568823861389375443 97351681296831567911618884222251141477322612331396 18606080373110348692660933940438416300326143449280 50821131575737727739821551522286509997662432587213 93393445902091662272905493493827178205126669021149 47192311380933822311224099588372246332501222323378 96895269025366263941267010317327864987170257149617 76105155492579857592045532468944687427025046397905 65326553194060999469787333810631719481735348955897

Digits $14,999,001$ to $15,000,000$ :

75161912582729034437123279749256311511925243956985 41466735069194815163837226073925151887751751659741 00622880726448602209456930414488539882981108512492 30626088375966783621649753412539683084922711342513 94953995693625441331401738133085848172315887473225 66862139251938540102249475575494947158395623512785 67033888824495551084462300472407612165952784386252 83059992302223284865934566262929748436827730812030 14434593689874259766415514412097984133998015934584 35393475650624323850160432731918805126406671871353 77555766214670931813151162879500509710551795152818 09093154481058044767364122166100032425098263166257 41730518220480715488224616563891344046934208103238 39903254029881746342496583186836947486194257533540 36331223838222392494056270856378033056213544686593 02986821714952808585949418676532291067339817684850 77576151785057277099880627370814385794117668763599 75814499149890314594098525960336377989988228138579 03954608500076180754880433958468619641092762653446 79645205263473393286074979323931503141172775669803 
Digits $19,999,001$ to $20,000,000$ :

24662421652199659486815804456870197576438951607697 86758526528445124126249995515004465281646092893016 37396198596248627116552469686381679679898926165214 19985145392716546108714664257998278750239431446690 24524827883001435830699295155565194378002452231513 03498450165135282534109758167508041457187906821950 98156889669401540575560430489547131781464796920586 99611799897126388736531564345333853581593559913668 62608486227029865668230856391322081859205243349223 41898466479821052634622968628766495150696262416056 24275201300452308788083860012754008114751496913646 62422297630443481605116791864334302662386921297850 27885235888942133721123400642720173755448172632485 38990548569368292370090889371435442648824207842546 28067400727949203553263884395310176843535902614634 76307233029969045465206192626213143248919480318684 24091340888618503237670440877047193079665717842568 49026897445701681738816789861189706430445720674936 81903857815020793466156644931359073005891342758785 95072447895232808191116291055801380049338634527644

Digits $24,999,001$ to $25,000,000$ :

64626376657788401626872035835150250932381126804132 24527774629670113871130617683224437149346115597163 91099108362268853888484703799982396604187954247350 36635859521304516872709809678948655853409228442863 24948936001342207955968740967092110719683856558205 30816048151902240856062148774123551023529985810792 74189214723685203602121713995138514107079374902532 54350785997288413483911434952219864948321330490074 60146435121254311259573947301142531184570914224080 72612210306331872567179327168155609249989038137333 66960257521334843154895361888436208731274888674781 18373984739313750077149269011462219615798047067514 35050981335283641909759090614464729227662129370246 47057090874450108027231969863517024941726518038367 32762891741863822149208539226376382907305941739639 07549588865849168186491743776278287261919660505923 92475738836587226649359524383297861404378228288281 73596312642574370611956801297356036342637793562761 38037507909491563108238168922672241753290045253446 07864115924597806944245511285225546774836191884322 
Digits $29,359,001$ to $29,360,000$ :

34192841788915229643368473881977698539005746219846 69525347577001729886543392436261840972591968259157 61107476294007303074005235627829787025544075405543 99895071530598162189611315050419697309728290606067 18890116138206842589980215445395753593792898823575 01412347486672046935635735777380648437308573291840 62108496330974827689411268675222975523230623956833 62631148916063883977661973091499155192847894109691 39612265329351195978725566764256462895375180907449 49363092921314127640888510170422584084744149319118 65755825721772836144977978766052285469047197596264 76680055360842209689517737135008611890452433015212 37693745702070338988940123376693961057269535278146 99719136307074643201853864071307997507974509883554 65961575782849747512645786441130845325323149405419 17263364899647912032878171893387317819324912382342 18648271763723022561720016348368584955658165112489 95446848720693621957797943429494640258419939089135 34266985232776239314365259670832026370250924776814 70490971424493675414330987259507806654322272888253

NAS Systems Division

NASA Ames Research Center

Mail Stop 258-5

Moffett Field, California 94035

1. D. H. BAILEY, "A high-performance fast Fourier transform algorithm for the Cray-2," J. Supercomputing, v. 1, 1987, pp. 43-60.

2. P. BeckmanN, A History of Pi, Golem Press, Boulder, CO, 1971.

3. A. Borodin \& I. MUNRo, The Computational Complexity of Algebraic and Numeric Problems, American Elsevier, New York, 1975.

4. J. M. BORWEIN \& P. B. BORWEIN, "The arithmetic-geometric mean and fast computation of elementary functions," SIAM Rev., v. 26, 1984, pp. 351-366.

5. J. M. BORWEIN \& P. B. BORWEIN, "More quadratically converging algorithms for $\pi$," Math. Comp., v. 46, 1986, pp. 247-253.

6. J. M. BORWEIN \& P. B. BORWEIN, $P i$ and the AGM-A Study in Analytic Number Theory and Computational Complexity, Wiley, New York, 1987.

7. R. P. BRENT, "Fast multiple-precision evaluation of elementary functions," J. Assoc. Comput. Mach., v. 23, 1976, pp. 242-251.

8. E. O. BRIGHAM, The Fast Fourier Transform, Prentice-Hall, Englewood Cliffs, N. J., 1974.

9. W. GOSPER, private communication.

10. EMIL GROSSWALD, Topics from the Theory of Numbers, Macmillan, New York, 1966.

11. G. H. HARDY \& E. M. WRIGHT, An Introduction to the Theory of Numbers, 5th ed., Oxford Univ. Press, London, 1984.

12. Y. Kanada \& Y. TAmura, Calculation of $\pi$ to $10,013,395$ Decimal Places Based on the Gauss-Legendre Algorithm and Gauss Arctangent Relation, Computer Centre, University of Tokyo, 1983.

13. D. KNUTh, The Art of Computer Programming, Vol. 2: Seminumerical Algorithms, AddisonWesley, Reading, Mass., 1981.

14. E. SAlamin, "Computation of $\pi$ using arithmetic-geometric mean," Math. Comp., v. 30, 1976, pp. 565-570.

15. D. Shanks \& J. W. Wrench, JR., "Calculation of $\pi$ to 100,000 decimals," Math. Comp., v. 16, 1962, pp. 76-99.

16. P. SWARztrauber, "FFT algorithms for vector computers," Parallel Comput., v. 1, 1984, pp. 45-64. 